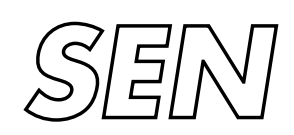

Software Engineering

$\int$ SEN Identifying aspects using fan-in analysis

A.M. Marin, A. van Deursen, L.M.F. Moonen

Report Sen-R0413 September 2004 
CWI is the National Research Institute for Mathematics and Computer Science. It is sponsored by the Netherlands Organization for Scientific Research (NWO).

$\mathrm{CWI}$ is a founding member of ERCIM, the European Research Consortium for Informatics and Mathematics.

CWI's research has a theme-oriented structure and is grouped into four clusters. Listed below are the names of the clusters and in parentheses their acronyms.

Probability, Networks and Algorithms (PNA)

\section{Software Engineering (SEN)}

Modelling, Analysis and Simulation (MAS)

Information Systems (INS)

Copyright (C) 2004, Stichting Centrum voor Wiskunde en Informatica

P.O. Box 94079, 1090 GB Amsterdam (NL)

Kruislaan 413, 1098 SJ Amsterdam (NL)

Telephone +31205929333

Telefax +31205924199 


\title{
Identifying aspects using fan-in analysis
}

\begin{abstract}
The issues of code scattering and tangling, thus of achieving a better modularity for a system's concerns, are addressed by the paradigm of aspect orientation. Aspect mining is a reverse engineering process that aims at finding crosscutting concerns in existing systems. This paper describes a technique based on determining methods that are called from many different places (and hence have a high 'fan-in') to identify candidate aspects in a number of open-source Java systems. The most interesting aspects identified are discussed in detail, which includes several concerns not previously discussed in the aspect-oriented literature. The results show that a significant number of aspects can be recognized using fan-in analysis, and that the technique is suitable for a high degree of automation.
\end{abstract}

1998 ACM Computing Classification System: D2.8, D2.3, D2.13

Keywords and Phrases: reverse engineering, aspect-oriented software development, software metrics

Note: Also appeared (without appendices) in the proceedings of the Working Conference on Reverse Engineering 2004 (WCRE), IEEE Computer Society Press, 2004. 


\section{Identifying Aspects using Fan-In Analysis}

\author{
Marius Marin \\ Software Evolution Research Lab \\ Delft University of Technology \\ The Netherlands \\ A.M.Marin@ewi.tudelft.nl
}

\author{
Arie van Deursen* \\ Software Evolution Research Lab \\ CWI \& Delft Univ. of Technology \\ The Netherlands \\ Arie.van.Deursen@cwi.nl
}

\author{
Leon Moonen \\ Software Evolution Research Lab \\ Delft Univ. of Technology \& CWI \\ The Netherlands \\ Leon.Moonen@computer.org
}

\begin{abstract}
The issues of code scattering and tangling, thus of achieving a better modularity for a system's concerns, are addressed by the paradigm of aspect orientation. Aspect mining is a reverse engineering process that aims at finding crosscutting concerns in existing systems. This paper describes a technique based on determining methods that are called from many different places (and hence have a high fan-in) to identify candidate aspects in a number of open-source Java systems. The most interesting aspects identified are discussed in detail, which includes several concerns not previously discussed in the aspect-oriented literature. The results show that a significant number of aspects can be recognized using fan-in analysis, and that the technique is suitable for a high degree of automation.

Note: To appear in the proceedings of the 11th Working Conference on Reverse Engineering (WCRE), IEEE Computer Society, 2004.
\end{abstract}

\section{Introduction}

Aspect-oriented programming (AOP) is a programming paradigm that addresses crosscutting concerns: features of a software system that are hard to isolate, and whose implementation is spread across many different modules. Wellknown examples include logging, persistence, and error handling. AOP captures such crosscutting behavior in a new modularization unit, the aspect, and offers (compile time) code generation facilities to weave aspect code into the rest of the system.

Aspect mining is an upcoming research direction aimed at finding aspect opportunities in existing, non-aspect oriented code. Once candidate aspects have been reverse engineered, they can be used for program understanding or reengineering purposes. Moreover, aspect mining techniques can be used by aspect novices to help them spot opportunities for using AOP techniques. Last but not least, an interesting side

${ }^{*}$ A. van Deursen received partial support from SENTER, project Ideals, hosted by the Embedded Systems Institute, Eindhoven, The Netherlands. effect of aspect mining research is that it increases our understanding of the nature of crosscutting concerns: it forces us to think about what good aspect opportunities are, and it helps us to find aspects that are beyond the canonical ones such as logging and error handling.

Aspect mining research, however, faces a number of challenges:

1. Which techniques can actually help us to find aspects in an automated way?

2. How can we decide that certain functionality should be implemented using an aspect? Since introducing an aspect is a design decision, this is likely to involve a trade off between alternatives. How can we make this trade off and how can we distinguish "good" aspect candidates from "bad" ones?

3. How can we evaluate the quality of an aspect mining technique, in terms of, e.g., precision and recall?

There are no simple answers to these questions. In order to obtain a better understanding of the problem domain, we decided to start out with an, in our opinion, simple and intuitive approach. The analysis we propose involves looking for methods that are called from many different call sites and whose functionality is needed across different methods, classes, and packages. Our approach aims at finding such methods by computing the fan-in metric for each method using the system's static call graph. It relies on the observation that scattered, crosscutting functionality that largely affects the code modularity is likely to generate high fan-in values for key methods implementing this functionality.

We adopt a systematic approach which consists of several steps suitable for a high degree of automation. The technique has turned out to be flexible and easy to combine with other techniques such as clone detection or slicing.

The analysis is focused on three open source systems so that the mined results can be shared and opened to a public debate. We report on the aspects found and explicitly discuss them, including several ones that were not previously investigated in the literature. 
The rest of the paper is structured as follows: In the next section we discuss related work. Section 3 describes our approach and illustrates the steps followed for one of the case studies. The most interesting concerns are reported for each case study in Sections 4-6. We discuss our results and the future work in Sections 7-8 and conclude in Section 9. More details on the case studies, a list of the selected concerns and a discussion of the methods that led to their identification are contained in the publicly available technical report [17].

\section{Related Work}

Although the topic of aspect mining is in its early stage of development, it is addressed by several research groups.

The identification of a crosscutting concern typically starts from a so-called seed: a method, interface or group of statements part of the concern's implementation. Once seeds have been identified, these can be expanded to full concern implementations by means of manual exploration, slicing, or other techniques. The various aspect mining approaches available to date differ in the techniques used for generating seeds and for extending seeds to full concerns, and in the level of automation provided in these steps.

Shepherd et al. [20] use clone detection based on the program dependence graph and the comparison of individual statement's abstract syntax tree representations, for mining aspects in Java source code. The tool built on this technique aims at a fully automatic mining process and is applied to two of the case-studies considered in this paper. Unfortunately, the list of the mined aspects is not reported.

Two clone detection tools implementing matching on tokens and abstract syntax trees respectively, are evaluated by [3] on an industrial $\mathrm{C}$ component. In this component, four dedicated crosscutting concerns were manually identified. The objective is to measure the concern coverage and to assess the suitability of clone detection for reconstructing these annotated aspects automatically.

Interfaces of which implementations are likely to be crosscutting are investigated in [22]. Their technique relies on string matching for the interface name, package membership and call relationships between the methods of the class implementing the interface.

The fan-in of modules was first defined by Henry and Kafura as number of locations from which control is passed into the module (e.g., calls to the module being studied) plus the number of global data accesses [11].

A number of frameworks and tools like Eclipse ${ }^{1}$ or FEAT [19] offer code navigation and understanding support, including the determination of a method's callers. Similar results, exposing relationships such as method calls, can be obtained using JQuery[12], an exploration tool with an underlying functional query language. Several mining dedicated

\footnotetext{
${ }^{1}$ http://www.eclipse.org/
}

tools, like AspectBrowser[8], Aspect Mining Tool [9] and $\mathrm{AMTEX}^{2}$, largely rely on lexical analysis - textual pattern matching. The latter two add a complementary type-based mining. All these tools need a starting seed from which the code to be explored further and based on this paper we believe fan-in analysis can provide such seeds. To the authors' knowledge there is no earlier work applying fan-in analysis for generating aspect mining seeds.

The suitability of several techniques, such as clone detection, slicing, dynamic analysis and concept and cluster analysis for the purpose of reverse engineering crosscutting concerns are discussed in [4]. We refer to [3, 4] for more detailed discussions of other aspect mining approaches.

\section{Aspect Identification Method}

Aspect mining can be conducted in two ways. The first is to take a top down approach, and search for code that implements well-known crosscutting concerns such as logging, persistence, error handling, etc. This calls for a catalog of typical aspects, collected from, e.g., text books or papers discussing aspect solutions to general design problems. Aspect mining research can then provide reconstruction approaches for each of the aspects in the catalog.

The second approach can be considered bottom up, and aims at analyzing the code for the symptoms resulting from a lack of proper aspect support in the language used. These symptoms are code scattering (the code for one concern is spread across the system) and code tangling (in order to implement one concern, the programmer needs to address other (crosscutting) concerns as well). Suitable techniques for identifying such symptoms may be clone detection (for scattering) or slicing (for untangling functionality), as discussed by [4]. Observe that the bottom up approach may be able to discover crosscutting concerns previously not recognized as suitable for an aspect solution.

In practice, aspect mining will be an opportunistic combination of these two approaches. The aspect mining method we propose here also combines both approaches, and originated from an (ongoing) attempt to create an explicit aspect catalog. Aspects in this catalog include generic ones such as logging and profiling [2, 7], as well as exception handling [16]. In addition, many design pattern implementations are candidates for an aspect implementation, as discussed by [10]. Finally, our catalogue contains aspect candidates that are tailored towards business applications such as transaction management, security [15], and J2EE design patterns.

The intuition gained from this study was that many known crosscutting concerns are implemented using one or more methods that exhibit a relatively high fan-in. In terms of AspectJ [2], the method would constitute (part of) the advice, and the call site would provide the context that would need to

\footnotetext{
2 http://www.eecg.utoronto.ca/ czhang/amtex/
} 


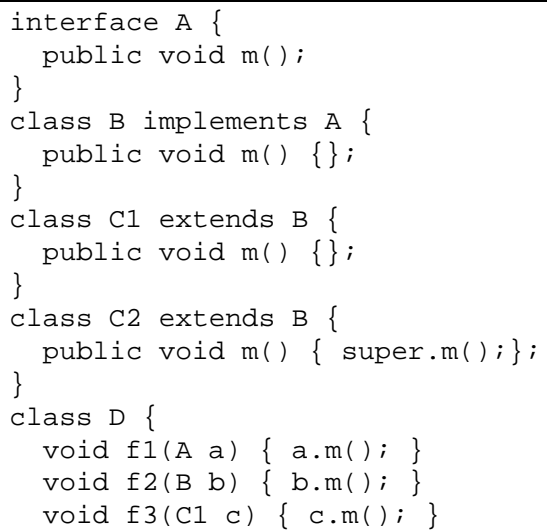

Figure 1. Various (polymorphic) method calls

\begin{tabular}{|l|l|c|}
\hline Method & Caller set & Fan-In \\
\hline A.m & $\{$ D.f1, D.f2, D.f3 $\}$ & 3 \\
\hline B.m & $\{$ D.f1, D.f2, D.f3, C2.m $\}$ & 4 \\
\hline C1.m & $\{$ D.f1, D.f2, D.f3 $\}$ & 3 \\
\hline C2.m & $\{$ D.f1, D.f2 $\}$ & 2 \\
\hline
\end{tabular}

Figure 2. Fan-in values for code in Figure 1

be captured using a point cut. Based on this observation, we decided to explore in depth how an analysis of methods with a high fan-in could lead us to candidate aspects in a number of open source Java case studies.

\subsection{The Fan-In Metric}

We define the fan-in of a method $m$ as the number of distinct method bodies that can invoke $m$. Because of polymorphism, one method call can affect the fan-in of several other methods. A call to method $m$ contributes to the fan-in of all methods refined by $m$ as well as to all methods that are refining $m$. Since this metric is fairly simple, except perhaps for the case of polymorphism, we do not provide a formal definition, but illustrate it using an example shown in Figure 1. Three different calls to polymorphic method $m$ are contained in class $D$. The resulting sets of callers and corresponding fan-in values are shown in Figure 2. Observe that the call in $f_{2}$ to $B$ 's $m$ contributes to the fan-in of $m$ in $B$ 's supertypes $(A)$ as well as its subclasses $\left(C_{1}\right.$ and $\left.C_{2}\right)$.

We have implemented the fan-in metric as a plug-in for the Eclipse Java IDE ${ }^{3}$. The plug-in makes use of standard Eclipse functionality - the search for references feature. Our plug-in computes the Fan-In metrics and reports the list of the callers for all the methods in the selected source code - project, package, class, etc. The output allows visualization of the callers and statistical reports.

\footnotetext{
3 http://www.eclipse.org/jdt/
}

\subsection{Identification Steps}

The analysis follows three consecutive steps:

Step 1. Automatic computation of the fan-in metric for all the methods in the targeted source code. The result is stored as a set of "method-callers" structures that can be sorted by fan-in value. This structure can be used to inspect the call sites and calling contexts of selected high fan-in methods.

Step 2. Filtering of the results of the first step:

- Restrict the set of methods to those having a fan-in above a certain threshold. We typically use a threshold of 10 , which tends to correspond to around $5 \%$ of the total number of methods.

- Filter getters and setters from this restricted set, based on the method's signature, in a first iteration, and its implementation, in a second iteration.

G(s-)etters on static fields are not eliminated because (as we shall see for example in the PETSTORE case in Section 4) these can be used in the Singleton design pattern, which may be a good candidate for an aspect representation [10].

- Filter utility methods, like toString (), collections manipulation methods, etc., from the remaining set.

Step 3. (Largely manual) Analysis of the remaining set of methods. The elements considered at this step are the callers and the call sites, the method's name and implementation, and the comments in the source code.

\subsection{Example}

In this section, we will describe the process of applying these steps to one of the case studies (PETSTORE) to illustrate the systematic approach. In later sections, we will discuss each of the case studies in more detail, putting more emphasis on the aspects that were discovered.

Java Pet Store Demo is a sample J2EE e-business application developed by SUN. ${ }^{4}$ It is intended as a demonstration of a real life Web application which allows customers to purchase via a web browser. In addition, it includes modules to perform administration tasks like sales statistics, orders and shipping management, etc.

Pet-store consists of approximately 17,000 non-comment lines of code. Our analysis shows a number of 1855 methods, including the abstract ones. The first step computes the fanin for each of these methods and distinguishes those having a high fan-in. Considering the results of the other case-studies as well as the ones for the present case, we have decided to analyze the methods with a fan-in value greater than 10 ,

\footnotetext{
4 http://java.sun.com/blueprints/, PETSTORE version 1.3.2.
} 




Figure 3. Fan-in distribution for PETSTORE.

\begin{tabular}{|l|c|c|c|c|c|}
\hline & seeds & non seeds & g(s-)etter & utility & Total \\
\hline \hline \multicolumn{5}{|l|}{ Fan-in $\in[10, \infty)$} \\
\hline no. & 7 & 1 & 7 & 5 & 20 \\
\hline$\%$ & 35.0 & 5.0 & 35.0 & 25.0 & 100.0 \\
\hline \hline \multicolumn{5}{|l|}{ Fan-in $\in[8,10)$} \\
\hline no. & 2 & 16 & 2 & 8 & 28 \\
\hline$\%$ & 7.1 & 57.2 & 7.1 & 28.6 & 100.0 \\
\hline
\end{tabular}

Figure 4. High fan-in methods in PETSTORE

which represents around $1 \%$ of the total $(1.08 \%$ - fig. 3$)$. The chosen reference value, of 10 , indicates a significant use of the method in terms of the number of calling methods, and also reduces substantially the number of subjects to be investigated. In a second iteration, we looked at the methods with a fan-in of 8 and 9 , which represent another $1.5 \%$ of the total. Statistics for the two fan-in sets are shown in Figure 4.

The second step performs a rough filtering of the high fan-in candidates. We were interested in both eliminating those methods likely to provide little information for the last step and in making sure that future automation of the filtering was possible. The filters for $\mathrm{g}(\mathrm{s}-)$ etters $(35 \%)$ and utility methods $(25 \%)$ together eliminated $60 \%$ of the candidates from the first step. Most of the getters are still related to aspects to be identified later on. The "utility" subset consist of tostring () methods and methods belonging to "util" classes, e.g. XMLDocumentUtils for building XML documents.

The remaining subset of $40 \%$ represents seed $(35 \%)$ and non-seed $(5 \%)$ candidates to be processed in the last step. Apart from one case which we decided not to include in the aspects category, all the other methods could be divided into several types of aspects: exception wrapping and business delegates, the Service Locator design pattern, contract enforcement and debugging functionality. All these concerns are discussed in detail in the PETSTORE section.

Applying the same steps to the methods with a fan-in of 8 and 9, we discovered only two new aspect seeds, one of them related to a new type - the Singleton design pattern.

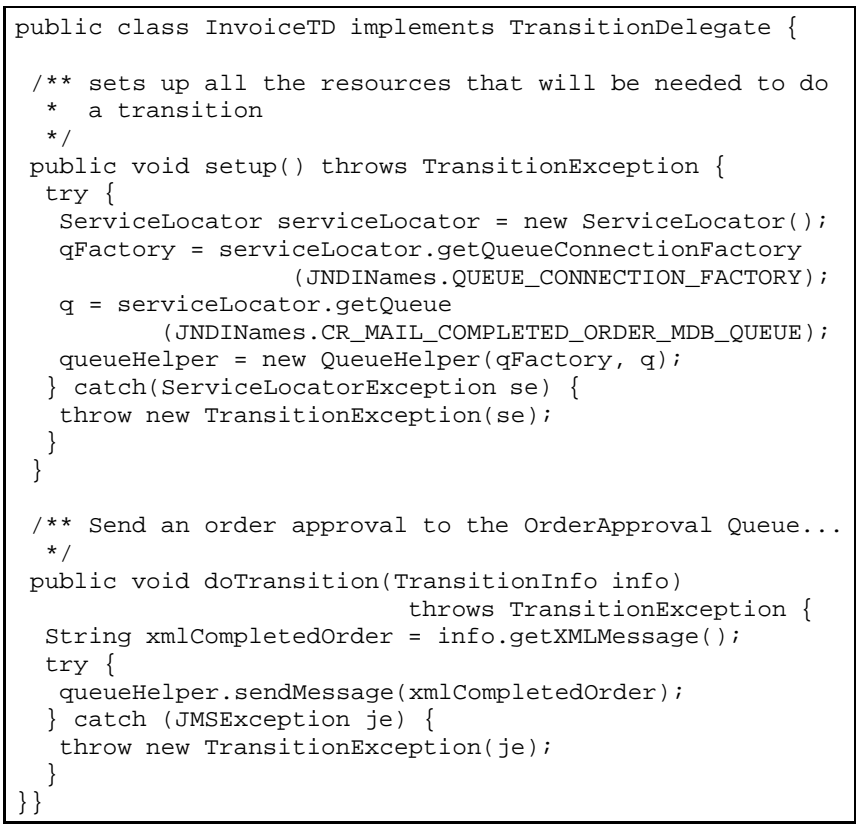

Figure 5. Error handling in PETSTORE

Two other methods, setup () and doTransition (..), that occur several times because of the polymorphical mechanism in the search procedure, were considered non-aspects as later discussed in the paper. The 15 instances reported for them cover around $50 \%$ of the second set of candidates. The utility methods have largely the same characteristics as the ones already outlined.

\section{Aspects Candidates in Pet Store}

Our analysis of the methods with fan-in above 10 and of 8 and 9 revealed the following cross cutting concerns.

Exception Wrapping and Business Delegates The majority of the aspect seeds are constructors for PETSTORE exceptions, with fan-in values ranging between 11 and 22. Figure 5 shows the example for the TransitionException case, which is thrown from 15 catch blocks in different classes and packages.

As in the InvoicetD class in the figure, most of the methods throwing the exception implement doTransition(..) and setup() declared by the TransitionDelegate interface. All the transition delegates handle exceptions related to the particular functionality and re-throw TransitionException. This mechanism is in fact part of the Business Delegate pattern [1], which aims at hiding the implementation details of a business service. The issue hidden in this case is the sort of exception that can be thrown by the actual implementation.

This mechanism is spread over many places, and a refactoring to aspects is in place. As discussed by Laddad [14], 
aspects can be used to isolate the exception handling and to wrap the original exception thrown by the underlying implementation in the new exception. This will result in improvements in code size, localization and clarity. Studies of exception handling refactoring [16] show a reduction of catch statements when using AOP of up to $95 \%$.

Service locators The method with highest fan-in value (30) belongs to the ServiceLocator class, which implements the J2EE pattern of the same name [1]. The intent of the pattern is to provide a single point of control to clients that need to locate and access a component or service in the business or integration tier. The common solution is to have a single instance of the service locator class for an application or, at least, for a tier and thus to have it implemented as a singleton. ${ }^{5}$ The delegates previously discussed are part of the locator's clients. They determine a high fan-in value for the locator access methods.

An analysis of the crosscutting nature of the J2EE patterns, including Service Locator and Business Delegate, was performed by [18]. The authors present a refactoring solution and analyze the advantages of an aspect-oriented implementation.

Contract Enforcement A method with a fan-in value of 27 is a constructor for the XMLDocumentException class. This exception is raised if the structure of the XML document does not comply with the expected structure. By examining the call sites, we were able to observe that 9 of them are fromDOM (Node) methods, all throwing the exception if a certain compound condition fails. It turns out that all complex conditions share a common check, which can be easily factored out as an aspect by means of before advice - giving rise to the contract enforcement concern as discussed in [2]. In this manner, the code will be better localized and new methods will be prevented from omitting the required checks.

Debug Information The XMLDocumentException class has a second constructor with a high fan-in. This constructor is (like for the business delegates) used as an exception wrapper. In addition to that, before being wrapped the exception at hand is written on the error output stream. This additional behavior (on top of the wrapping) can be added as another aspect, which indicates which exception should be printed before being wrapped. The aspectization of printing such debugging information is desirable to ensure a common debugging strategy and to isolate the concern that otherwise is crosscutting.

\footnotetext{
${ }^{5}$ PETSTORE contains two different service locators, one for JMS and one for EJB components. The former is indeed a singleton (as required in [1]), but the other is not. It does contain a private static fi eld, but this is not used. The fact that this service locator is not implemented as a singleton is an error in the PETSTORE implementation —an error that can be easily fi xed by superimposing the AOP singleton solution on top of it.
}

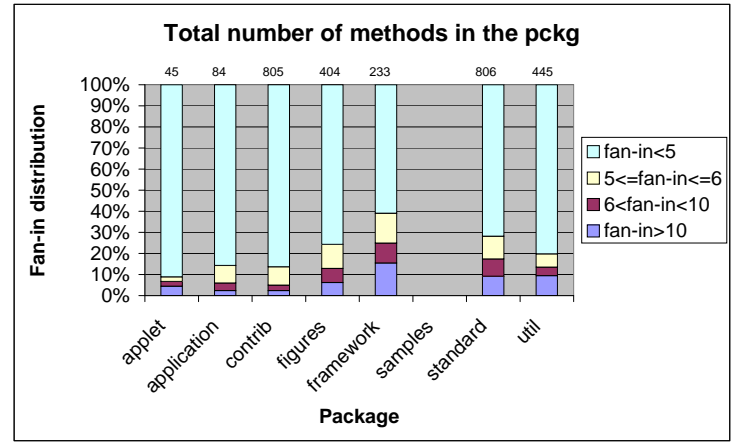

Figure 6. Fan-in statistics for JHOTDRAW.

False Alarm The one case considered as non-aspect in the first set of candidates is an XMLDBHandler constructor with a fan-in value of 10 . The callers are setup (..) methods in classes that populate the associated database tables with data from XML files. The setup (..) implementations are only slightly different: return an instance of an anonymous inner class extending XMLDBHandler that is an XML filter. Because all the callers are well localized in a single package and there is only one populate (..) method that triggers the whole process at a client's request, we decided to ignore the candidate.

Lower Fan-In Values The set for the fan-in values of 8 and 9 comprises, because polymorphism, 15 instances of the same two methods, doTransition (.. ) and setup (), declared in the TransitionDelegate interface. As all transition delegates implement this only interface as their primary concern, the two methods were not considered aspect seeds.

Singleton Two of the interesting methods with fan-in of 8 and 9 are related to the Singleton pattern, which is used for one of the ServiceLocator classes and the StatusBar. The high fan-in method is get Instance () which yields the reference to the unique instance. Aspect oriented refactorings for the design patterns from [5] have been proposed by [10] and showed improvements in terms of the modularity properties for most of the cases, including Singleton.

\section{JHotDraw}

JHotDraw $^{6}$ is an application framework for two-dimensional graphics. It was designed as an exercise to show a good use of design patterns [5]. The version analyzed is JHotDraw $5.4 \mathrm{~b} 1$ of approximately 18,000 non-comment lines of code.

Figure 6 shows how the fan-in values for methods are distributed across the various packages of JHOTDRAW. In terms of methods with high fan-in values, above 10, two

\footnotetext{
${ }^{6}$ http: // jhotdraw. org/, version 5.4b1
} 


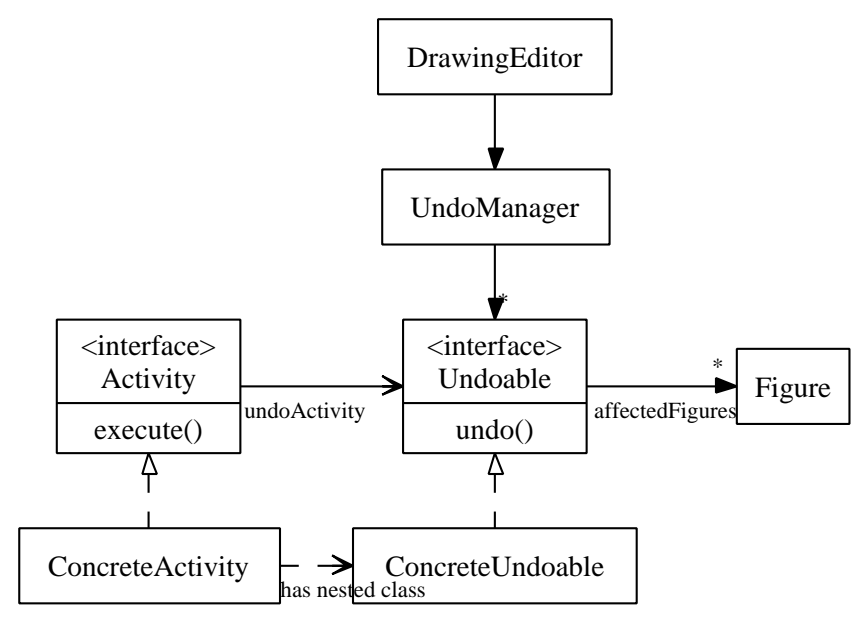

Figure 7. Participants for undo in JHOTDRAW.

packages show the top relative and absolute values, respectively: "framework", which contains the classes and the interfaces that define the project's framework, and "standard", which provides the default implementation for the framework classes. The latter is the project's largest package.

For each of the two packages, our filters eliminated around half of the methods with top fan-in values. We were rather cautious not to eliminate too many methods. The only methods designated as "utility" are enumerations manipulators (e.g., FigureEnumerator.hasNextFigure()/nextFigure()).

\subsection{The Undo Concern}

In the top of the list of methods with highest fan-in, there are several methods that contain the word "undo" in their names, or that are part of a class whose name contains "undo". An undo in a graphical editor is clearly a concern that cuts across many features and activities.

A (somewhat simplified) representation of the participating classes in the JHOTDRAW undo implementation is given in Figure 7. JHOTDRAW offers various sorts of activities, which are contained in a class hierarchy. Examples of concrete activities include handling font sizes, triangle rotation, or image rotation.

The interface Undoable encapsulates the notion of undoing an action, for which it provides the undo method. Each class implementing a concrete activity that can be undone, defines a static nested class conforming to this Undoable interface. The nested class knows how to undo the given activity, and has access to all the details of the activity that may be needed for this. Whenever the activity modifies its state, it also updates fields in its associated undo-activity needed to actually perform the undo. In addition to that, a list of affected figures is maintained, whose state is also affected if the activity must be undone.

In JHOTDRAW, there are 22 activities that can be undone, causing the undo concern to be spread over these classes. This, in turn, leads to a high fan-in for the methods of, for example, Undoable, which helped us to identify this crosscutting concern.

Refactoring undo using aspects takes a number of steps. First, the existing activities can be extended with an association to their undoables by means of a separate introduction (through an AspectJ intertype declaration). Second, existing operations should be extended with functionality to keep track of the old state so that the action can be undone. These existing operations can be captured using a pointcut, and then the updates can be contained in advice code. The various nested classes containing the undoable activities can also be added by means of introductions / intertype declarations. ${ }^{7}$ The overall effect of this aspectization is that the key functionality remains separated from maintaining the undo state, making both easier to comprehend and modify.

In spite of the fact that "Undo" is a concern that is a natural candidate for an aspect-oriented solution, textbooks on AOP (such as $[7,2,15]$ ) do not discuss using aspects for undo functionality. On the Codehaus electronic forum, Jon Tirsen provides an interesting generic solution for the undo problem [21]. Tirsen defines a Command class, and provides a point cut capturing any field access occurring in the command's context. He then uses reflection to store the field's old values in the command, so that a generic undo is made possible.

\subsection{Persistence}

Another cross cutting concern that pops out clearly through a high fan-in is persistence. Persistence and resurrection of figures is performed by methods inherited from the Storable interface. The two are pair concerns: the Figure classes implement the methods to write/read themselves to/from a Storableoutput/Input object, which is basically a specialized output/input stream. We will discuss only the persistence concern, resurrection being just similar.

Persistence is a crosscutting concern spread over 36 classes. The concrete figures implement the Storable interface that encapsulates the concern. The Storable is what we call a secondary interface - an interface that does not define the primary role of the implementing class but only adds some functionality. The methods ensuring the persistent behavior create a high fan-in for several members of the StorableOutput class.

A refactoring solution, using the features of the AspectJ language, would use introduction to isolate the persistence concern from the figure classes. Better modularity properties can be achieved by having all the code relating to the storable functionality in the aspect construct. The figures will implement no persistence-specific code but will be extended with

\footnotetext{
7 The present version of AspectJ does not yet support introducing inner and static nested classes.
} 




Figure 8. (Simplified) execute method in JHотDRAW exhibiting tangling.

this functionality through inter-type declarations.

\subsection{Observers in JHotDraw}

The Observer is well known as a good candidate for aspect refactoring, due to the roles defined in the pattern's context that crosscut the participant classes' logic. The elements indicative of the Observer design pattern are the methods used to at(de-)tach the observers to the subject participant, to notify the observer participants of the subject's changes and to update them accordingly. We expect the pattern to be suitable for fan-in recognition through these key-elements. Observers that register themselves as listeners to the subject's changes increase the metric's value for the attach method. In turn, the notify method will be called by every subject's modifier in order to trigger an update operation for the observers.

After these key methods of the Observer pattern have been identified by means of fan-in analysis, existing exploration tools and methods can be used to understand the pattern-aspect implementation and its extent. Although the result of this identification method depends on the number of the registered observers and subject changers, our experiments show good results in finding Observer's elements by applying the fan-in metrics.

The set of the methods with high fan-in values comprises a number of seeds for Observer instances such as the changed () and add/removeFigureChangeListener (..) methods in Figure classes. The value for the changed () method is as high as 37 . Once defined the Figure's subject role in the pattern context, an analysis of the call sites will lead to the identification of the other participants.

Matching on the naming conventions used in the identified Observer led to another instance of the pattern (with a somewhat lower fan-in). Thus, fan-in analysis provides initial seeds and application understanding, which then can be used by complementary techniques to identify further cross cutting concerns.

\subsection{Other Concerns}

JHOTDRAW being an exercise in the use of design patterns, it is not surprising that JHOTDRAW has a high pattern density. Here we analyze which patterns have a cross cutting nature in JHOTDRAW, and how they can be found through fan-in analysis. Hannemann et al. provide a general discussion how a number of the classical design patterns could benefit from an AOP implementation [10].

The structure of several design patterns and the associated collaborations can support their fan-in identification. It is yet difficult to turn this identification into a general rule because, most of the time, it is project size and context dependent whether the design pattern has a key role in the design or just provides an isolated solution.

With high fan-in values reported for Composites' children manipulation methods (e.g., add(Figure) and figures () for standard drawings and thus, for composite figures, and abstract figures) the pattern was readily recognizable.

Singleton classes have been found through the instance()like methods, returning the unique instance of the class (see also Section 4).

Command's execute() operation is invoked by multiple clients: The execute () method in the AbstractCommand class is reported with a fan-in value of 24 . There are 10 implementations of this method: An example implementation is provided in Figure 8. The execute method is a seed for multiple aspects:

- Each execute implementations starts by checking a common pre-condition, throwing an exception if it does not hold. This is a contract enforcement aspect as discussed in [2].

- Most execute implementations conclude with a check if the figure has been changed and a corresponding refresh. This is a Providing consistent behavior aspect as discussed in [2].

Factoring these (as well as the undo functionality) out of the code in Figure 8 would leave the execute method with just its core functionality, which is an implementation of the cut operation by means of a copy and a delete operation.

The instances of the Decorator and Adapter are more difficult to recognize, mainly because unlike the other patterns already discussed, there is not a key method that strongly connotes the link with the pattern. The manual analysis relied more in these cases on the information obtained from naming conventions and the comments in the source code. However, the request forwarding from the decorator/adapter object to the component/adaptee participants could also be considered (e.g., containsPoint (), includes (), etc. in DecoratorFigure all with fan-in values greater than $10)$. 
The Providing consistent behavior concern discussed in [2] aims at ensuring that certain functionality is implemented uniformly across a large set of operations. In the Storableoutput class, the various printing operations to the PrintWriter object are followed by a call to the space() method which simply prints a space to the same object. The method has a high fan-in value, all the calls being from inside the class. It is a good example of crosscutting at class level and also an easy-to-imagine refactoring that will add the space() call after the print(..)'s execution.

Among the other reported candidates the displayBox() method for Figure and Handle is worth mentioning. It returns the object's display box and is overloaded for figures for changing a figure's display box. The examination of the call sites showed many draw (Graphics) callers in handle classes and in similar calling contexts. Despite that, we could not see much benefits from a refactoring. Although the method is reported in several instances, we think it supports the class's main functionality and is not crosscutting.

\section{Tomcat/Catalina}

TOMCAT is a free, open-source implementation of Java Servlet and JavaServer Pages technologies developed under the Jakarta project at the Apache Software Foundation. Catalina is the servlet container portion of Tomcat. ${ }^{8}$ Our analysis is concentrated on the Catalina component. It consists of approximately 90,000 non-commented lines of code.

\subsection{Overview of the results}

Among the candidates mined, logging is one of the crosscutting concerns which is often acknowledged to exist in the Tomcat implementation. The detection of logging by means of fan-in analysis is also not surprising since it is often implemented by means of calls to a commmon method.

The other results found include context passing and contract enforcement as well as design patterns, such as $\mathrm{Ob}$ server, Composite, Chain of responsibility, Command, Singleton or Adapter. These candidate aspects all have characteristics similar to those discussed for the other case-studies so we will omit them for conciseness.

The next section describes the new Lifecycle concern that we discovered in TOMCAT. We are not aware of any previous discussion of this concern in (AOP) literature.

\subsection{Lifecycle}

Lifecycle is a common interface for the life cycle methods of Catalina components. The documentation states that is intended to provide consistent start and stop mechanisms

\footnotetext{
http://jakarta.apache.org/tomcat/tomcat-5. 0-doc/catalina/docs/api/index.html, v. 5.0.24
}

and should be implemented apart from the interfaces that define the functionality supported by each of the components. This makes Lifecycle a secondary interface - it adds new, supplementary capabilities to the core logic of the implementing classes. In the case investigated, the Lifecycle interface is implemented by about 20 classes.

Implementors of the Lifecycle interface are subjects in the context of the Observer design pattern and the start () and stop () methods have to provide the notifying functionality. In addition, these two methods are part of a contract enforcement scheme: The start () operation has to be called before any public method of the component, while stop () terminates the object's use and should be the last call for a component's instance.

The Lifecycle concern can be seen as a generalization of the use of stop() methods to remediate Java's expensive finalization mechanism [23,6]. Those methods take care of cleaning up the object's resources inside the program code to avoid the overhead of having finalizers but will result in crosscutting for the object's clients.

The Lifecycle concern is complex and although aspect oriented solutions have been presented for some parts of it, a complete refactoring solution remains an open issue. One of the problems with proposing a full solution is that the type of contract enforcement needed by the concern cannot be expressed in a pointcut based aspect language like AspectJ (because it requires specifying "before accessing any public methods of class" and "after last use of class").

\section{Discussion}

The statistical results show that more than one third of the methods with high fan-in are seeds that lead to aspects. Using filtering, we can automatically remove $60 \%$ of the remaining two thirds, leaving almost only seeds of interest.

We can distinguish three situations in which a high fan-in value indicates the presence of crosscutting concerns (in no particular order):

1. The high fan-in method is a key element of the aspect implementation, such as the output method for logging, tracing or debugging functionalities. In such a case, the refactoring to aspects will be driven by the presence of the seed-method calls.

2. The crosscutting implementation is spread over the system and relies on common functionality and the high fan-in method is part of this functionality. In this case, the call sites give the insight into the aspect, e.g., the persistence aspect previously discussed.

3. Some design patterns with a crosscutting structure can lead to high fan-in values when they are given a central role in the project design. This can, for example, 
happen to the observer manipulation methods that are associated with the subject role in the Observer design pattern. When such methods are found, it strongly implies that the pattern was applied.

We can make a number of observations concerning the results of our fan-in based analysis:

The type of concerns identified - The fact that we were able to find similar aspects in various case studies suggests that their identification is not accidental. The results contains various crosscutting concerns that are discussed in the literature, including those that stood at the origins of AOSD. In addition, we have identified a number of new aspects, such as Undo and Lifecycle. Given the variety in the case studies, we feel that these results can also be achieved for other cases.

False positives and negatives - We feel that fan-in based analysis is especially good at identifying crosscutting concerns that have a relatively large "footprint" in the source code (since that generally results in higher fan-in). There are both positive and negative sides to this: On the one hand, the aspects identified this way are likely to be those that significantly influence the modularity of the source code and thus most appropriate for refactoring. On the other hand, aspects with a smaller footprint, such as a design pattern providing an isolated solution, could be missed because the number of calls is below the threshold. In any case, the (unfiltered) set of results of fan-in analysis can provide useful information for other mining techniques. Once aware of the potential presence of an aspect, other approaches can be followed to assure or refute it's existence.

It is difficult to discuss false negatives in more detail since it would require a report of all the aspects that could be found in the case studies considered. Such reports are not available at the moment, and we consider our work to be a first step towards a common benchmark for various aspect mining techniques being developed. To promote such a common benchmark, we are in the process of setting up a (wiki-based) web forum ${ }^{9}$ where aspect mining researchers can exchange and discuss aspect candidates found in (open source) software systems. We will populate this forum with the results discussed in this paper.

Steps towards automation - The three steps followed in our approach can be automated to a large extend: The first step (Fan-In computation) is already automated as an Eclipse plugin. However, because of the influence of polymorphism during the search for references, in our current implementation the same method could be reported as having high fan-in for different instances. We do not consider this a major issue as it could be repaired by a more in-depth analysis. A more serious limitation could be fan-in aggregation - two methods with a relatively high fan-in, but not enough to be individually reported, both calling a third method for which will create a high aggregate fan-in. None of these methods

\footnotetext{
${ }^{9}$ http://swerl.tudelft.nl/amr/
}

will be reported. An experiment that considers the aggregation would be interesting to perform.

The second (filtering) step is this initial investigation can both be further automated and improved. We generally only filter the simple $\mathrm{g}(\mathrm{s}-)$ etters (returning or setting a member field value) as they are not likely to offer enough information for further exploration. However, the filtering based on methods' signatures, although substantially reducing the set to be manually investigated, could result in false negatives.

The automation of the last step will mainly consist in applying complementary techniques such as clone detection and concept analysis and use their results to both augment and reinforce our own results.

Language independence - An additional case study with fan-in based aspect mining on the 19 KLOC industrial C component that was used in [3] to evaluate clone detection based aspect mining, performed encouragingly well. The main methods in the implementation of the tracing and logging crosscutting concerns have reported fan-in values of 200 and 400, respectively. The metric's value was lower than 10 for the other methods.

\section{Future work}

One of our goals is achieving a considerable degree of automation. To that end, we are going to extend our mining process as previously discussed. A longer term plan is an aspect mining tool that combines several analysis techniques to achieve a higher degree of completeness and precision.

Fan-in based analysis turns out to be flexible and very suitable for combination with other techniques, such as clone detection or concept analysis. We have just finished a study were we applied the CCFinder [13] clone detection tool to the JHotDraw case study. The clone classes reported in this study as aspect candidates pointed to the same persistence and resurrection aspects identified by fan-in analysis. This result gives us the anecdotal evidence to further explore the combination of these two techniques.

The call site of a high fan-in method can represent a cross cutting concern, but typically the concern is larger than just the call itself, involving setting up appropriate objects and checking relevant conditions. We are investigating how slicing like techniques can be used to find more complete concerns based on initial seeds identified.

Finally, there is a relation that needs to be investigated between the aspectizable interfaces as described in [22] and the methods with a high fan-in value declared by such interfaces. A starting point for such an investigation could be the Undoable interface of JHOTDRAW where a number of methods have reported a high fan-in. 


\section{Concluding Remarks}

In this paper we analyzed three open source Java systems in significant detail, searching for opportunities to use aspect oriented techniques in them. Our search was guided by the fan-in value for each method, following the simple rule that a method with a high fan-in is likely to represent functionality that is required across the application. The key contributions of this paper are the systematic approach used to identify the aspects (described in Section 3) and the discussion of the aspects actually found (in Sections 4-6).

The primary objective of our analysis was to explore the feasibility of reverse engineering aspects from object oriented code. The results of our analysis can be used in the following ways:

- The various examples of candidate aspects can be used to come up with new techniques that can reverse engineer these aspects (semi)automatically.

- The aspects we identified manually can be used in order to validate the effectiveness of new aspect mining techniques.

- The technique can be either used stand-alone or in combination with other techniques.

An interesting side effect of our analysis is that we found a number of opportunities for using aspect oriented techniques, such as the undo and life cycle functionality discovered in JHOTDRAW and TOMCAT, that were not previously described in the aspect-oriented literature.

Last but not least, the nature of the case studies considered provide an assessment that crosscutting is a problem even in the well designed projects. The number of aspects we were able to find enforces the claims that cross cutting is inherent in an $\mathrm{OO}$ system. The fan-in analysis, besides of identifying them can give a measure of their impact.

\section{References}

[1] D. Alur, J. Crupi, and D. Malks. Core J2EE Patterns. Sun Microsystems, Inc., USA, 2003.

[2] The AspectJ Team. The AspectJ Programming Guide. Palo Alto Research Center, 2003. Version 1.2.

[3] M. Bruntink, A. van Deursen, R. van Engelen, and T. Tourwé. An evaluation of clone detection techniques for identifying cross-cutting concerns. In Proceedings International Conference on Software Maintenance (ICSM 2004). IEEE Computer Society, 2004

[4] A. van Deursen, M. Marin, and L. Moonen. Aspect mining and refactoring. In Proceedings of the First International Workshop on REFactoring: Achievements, Challenges, Effects (REFACE03). University of Waterloo, Canada, 2003.
[5] E. Gamma, R. Helm, R. Johnson, and J. Vlissides. Design Patterns: Elements of Reusable Object-Oriented Software. Addison-Wesley, 1994.

[6] B. Goetz. Garbage collection and performance. IBM developersWorks articles, January 2004. www-136.ibm. com/ developerworks/java/.

[7] J. D. Gradecki and N. Lesiecki. Mastering AspectJ - Aspect Oriented Programmingin Java. Wiley Publishing, Inc., Indianapolis, Indiana, 2003.

[8] W. G. Griswold, Y. Kato, and J. J. Yuan. Aspectbrowser: Tool support for managing dispersed aspects. Technical Report CS99-0640, University of California, San Diego, 3, 2000.

[9] J. Hannemann and G. Kiczales. Overcoming the prevalent decomposition of legacy code. In Proc. Workshop on Advanced Separation of Concerns. IEEE, 2001.

[10] J. Hannemann and G. Kiczales. Design pattern implementation in Java and AspectJ. In Proceedings of the 17th Annual ACM conference on Object-Oriented Programming, Systems, Languages, and Applications (OOPSLA), pages 161173. ACM Press, 2002.

[11] S Henry and K Kafura. Software structure metrics based on information fbw. IEEE Transactions on Software Engineering, 7(5):510-518, 1981.

[12] D. Janzen and K. De Volder. Navigating and querying code without getting lost. In Proc. 2nd Int. Conf. on AspectOriented Software Development (AOSD), pages 178-187. ACM Press, March 2003.

[13] Toshihiro Kamiya, Shinji Kusumoto, and Katsuro Inoue. Ccfi nder: a multilinguistic token-based code clone detection system for large scale source code. IEEE Trans. Softw. Eng., 28(7):654-670, 2002.

[14] R. Laddad. Aspect-oriented refactoring. WwW . theserverside. com, December 2003.

[15] R. Laddad. AspectJ in Action - Practical Aspect Oriented Programming. Manning Publications Co., 2003.

[16] M. Lippert and C.V. Lopes. A study on exception detection and handling using aspect-oriented programming. In Proceedings of the 22nd international conference on Software engineering, pages 418-427. ACM Press, 2000.

[17] M. Marin, A. van Deursen, and L. Moonen. Identifying aspects using fan-in analysis. Technical Report SENR0413, CWI, 2004. www.cwi.nl/ftp/CWIreports/ SEN/SEN-R0413.pdf.

[18] T. Murali, R. Pawlak, , and H. Younessi. Applying aspect orientation to J2EE business tier patterns. In Proc. of the 3rd AOSD Workshop on Aspects, Components, and Patterns for Infrastructure Software. University of Lancaster, UK, 2004.

[19] M.P. Robillard and G.C. Murphy. Concern graphs: Finding and describing concerns using structural program dependencies. In 24th International Conference on Software Engineering (ICSE). ACM press, 2002.

[20] D. Shepherd, E. Gibson, and L. Pollock. Design and evaluation of an automated aspect mining tool. In Proceedings Mid-Atlantic Student Workshop on Programming Languages and Systems, 2004. 
[21] J. Tirsen. Undo in AspectJ. Codehause Jutopia discussion forum, April 25 2004. blogs. codehause.org.

[22] P. Tonella and M. Ceccato. Migrating interface implementation to aspect oriented programming. In Proceedings International Conference on Software Maintenance (ICSM 2004). IEEE Computer Society, 2004.

[23] P. Vickers. Why fi nalizers should (and can) be avoided. IBM developersWorks articles, March 25 2002. www-136. ibm . com/developerworks/java/. 


\section{A. Case Studies Overview}

\begin{tabular}{|c|c|c|c|c|}
\hline Project name & version & url & NCLOC & \#methods \\
\hline JHotDraw & $5.4 \mathrm{~b} 1$ & http://ihotdraw.org & 18,000 & 2822 \\
\hline PetStore & 1.3 .2 & $\begin{array}{c}\text { http://java.sun.com/blueprints } \\
\text { http://jakarta.apache.org/tomcat }\end{array}$ & 17,000 & 1855 \\
\hline Tomcat & 5.0 .24 & $\begin{array}{l}\text { /tomcat-5.0- } \\
\text { doc/catalina/docs/api// }\end{array}$ & 90,000 & 4166 \\
\hline
\end{tabular}




\section{B. Statistics for JHotDraw}

The statics of the methods with a fan-in value greater than 10 , organized by

\begin{tabular}{|c|c|c|c|c|c|c|}
\hline \multicolumn{2}{|l|}{ Package } & seeds & prome & (s-)etter & utility & Total \\
\hline applet & no. & 1 & 0 & 1 & 0 & 2 \\
\hline & $\%$ & $50.00 \%$ & $0.00 \%$ & $50.00 \%$ & $0.00 \%$ & $100.00 \%$ \\
\hline application & no. & 1 & 0 & 1 & 0 & 2 \\
\hline & $\%$ & $50.00 \%$ & $0.00 \%$ & $50.00 \%$ & $0.00 \%$ & $100.00 \%$ \\
\hline contrib & no. & 0 & 13 & 6 & 0 & 19 \\
\hline & $\%$ & $0.00 \%$ & $68.42 \%$ & $31.58 \%$ & $0.00 \%$ & $100.00 \%$ \\
\hline figures & no. & 2 & 14 & 6 & 0 & 22 \\
\hline & $\%$ & $9.09 \%$ & $63.64 \%$ & $27.27 \%$ & $0.00 \%$ & $100.00 \%$ \\
\hline framework & no. & 12 & 8 & 14 & 2 & 36 \\
\hline & $\%$ & $33.33 \%$ & $22.22 \%$ & $38.89 \%$ & $5.56 \%$ & $100.00 \%$ \\
\hline standard & no. & 25 & 12 & 26 & 11 & 74 \\
\hline & $\%$ & $33.78 \%$ & $16.22 \%$ & $35.14 \%$ & $14.86 \%$ & $100.00 \%$ \\
\hline util & no. & 11 & 1 & 18 & 12 & 42 \\
\hline & $\%$ & $26.19 \%$ & $2.38 \%$ & $42.86 \%$ & $28.57 \%$ & $100.00 \%$ \\
\hline Total & no. & 52 & 48 & 72 & 25 & 197 \\
\hline & $\%$ & $26.40 \%$ & $24.37 \%$ & $36.55 \%$ & $12.69 \%$ & $100.00 \%$ \\
\hline
\end{tabular}




\title{
C. Concerns and Fan-In Values for JHoTDRAW
}

\author{
List of the seed methods for JHotDraw
}

Note: In some cases it was difficult to make explicit the difference between the "contract enforcement" and "providing consistent behavior" aspects, as described in [2]. For this reason, the two generic aspects are both indicated for some of the seeds in the list.

\begin{tabular}{|c|c|c|}
\hline Qualified name (CH.ifa.draw. ${ }^{\star}$ ) & Fan-in val. & Concern \\
\hline applet.DrawApplet.toolDone() & 16 & Consistent behavior \\
\hline application.DrawApplication.toolDone() & 18 & Consistent behavior \\
\hline figures.NullFigure.includes(Figure) & 13 & Composite \\
\hline figures.TextFigure.changed() & 36 & Observer \\
\hline framework.DrawingEditor.toolDone() & 20 & Consistent behavior \\
\hline framework.DrawingView.add(Figure) & 10 & Composite \\
\hline framework.DrawingView.checkDamage() & 28 & Change monitoring \\
\hline $\begin{array}{l}\text { framework.DrawingView.selectionCount() } \\
\text { framework.Figure.addFigureChangeListener( }\end{array}$ & 16 & Consistent behavior \\
\hline FigureChangeListener) & 11 & Observer \\
\hline framework.Figure.changed() & 36 & Observer \\
\hline framework.Figure.figures() & 24 & Composite \\
\hline framework.Figure.includes(Figure) & 14 & Composite \\
\hline framework.Figure.listener() & 21 & Observer \\
\hline framework.Figure.removeFigureChangeListener( & & \\
\hline FigureChangeListener) & 10 & Observer \\
\hline framework.Figure.willChange() & 25 & Observer \\
\hline framework. Handle.owner() & 37 & Adapter \\
\hline $\begin{array}{l}\text { standard.AbstractCommand.execute() } \\
\text { standard.AbstractFigure.addFigureChangeListener( }\end{array}$ & 24 & $\begin{array}{c}\text { Command } \\
+ \text { Contract enforcement }\end{array}$ \\
\hline FigureChangeListener) & 11 & Observer \\
\hline standard.AbstractFigure.changed() & 37 & Observer \\
\hline standard.AbstractFigure.figures() & 24 & Composite \\
\hline standard.AbstractFigure.includes(Figure) & 14 & Composite \\
\hline standard.AbstractFigure.removeFigureChangeListener( & & \\
\hline FigureChangeListener) & 10 & Observer \\
\hline standard.AbstractFigure.willChange() & 25 & $\begin{array}{c}\text { Observer } \\
\text { Consistent behavior/ }\end{array}$ \\
\hline standard.AbstractTool.AbstractTool(DrawingEditor) & 15 & $\begin{array}{l}\text { Contract enforcement } \\
\text { Consistent behavior/ }\end{array}$ \\
\hline standard.AbstractTool.activate() & 10 & $\begin{array}{l}\text { Contract enforcement } \\
\text { Consistent behavior/ }\end{array}$ \\
\hline standard.AbstractTool.deactivate() & 14 & Contract enforcement \\
\hline standard.AbstractTool.mouseDown(MouseEvent, int, int) & 15 & Consistent behavior \\
\hline standard.CompositeFigure.includes(Figure) & 14 & Composite \\
\hline standard.DecoratorFigure.containsPoint(int, int) & 15 & Decorator \\
\hline standard.DecoratorFigure.displayBox() & 50 & Decorator \\
\hline standard.DecoratorFigure.includes(Figure) & 13 & Composite + Decorator \\
\hline $\begin{array}{l}\text { standard.DecoratorFigure.moveBy(int, int) } \\
\text { standard.DeleteCommand.DeleteCommand( }\end{array}$ & 13 & Decorator \\
\hline String, DrawingEditor) & 12 & Command \\
\hline standard.NullDrawingView.checkDamage() & 23 & Change monitoring \\
\hline
\end{tabular}




\section{Concerns and Fan-In Values for JHotDRAW- cont.}

\begin{tabular}{|c|c|c|}
\hline Qualified name (CH.ifa.draw.*) & Fan-in val. & Concern \\
\hline & & Consistent behaviour/ \\
\hline standard.NullDrawingView.clearSelection() & 30 & Contract enforcement \\
\hline standard.NullDrawingView.selectionCount() & 13 & Consistent behavior \\
\hline standard.StandardDrawing.add(Figure) & 13 & Composite \\
\hline standard.StandardDrawingView.add(Figure) & 10 & Composite \\
\hline standard.StandardDrawingView.checkDamage() & 28 & $\begin{array}{l}\text { Change monitoring } \\
\text { + Observer-notifier } \\
\text { Consistent behaviour/ }\end{array}$ \\
\hline standard.StandardDrawingView.clearSelection() & 31 & Contract enforcement \\
\hline standard.StandardDrawingView.selectionCount() & 16 & Consistent behavior \\
\hline util.Storablelnput.readlnt() & 22 & Persistence \\
\hline util.Storablelnput.readStorable() & 20 & Persistence \\
\hline util.Storablelnput.readString() & 10 & Persistence \\
\hline util.StorableOutput.writelnt(int) & 21 & Persistence \\
\hline util.StorableOutput.writeStorable(Storable) & 18 & Persistence \\
\hline util.StorableOutput.writeString(String) & 10 & Persistence \\
\hline util.Undoable.isRedoable() & 26 & Undo \\
\hline util.Undoable.isUndoable() & 11 & Undo \\
\hline util.UndoableAdapter.undo() & 24 & Undo \\
\hline util.UndoableAdapter.UndoableAdapter(DrawingView) & 26 & Undo \\
\hline util.UndoableCommand.UndoableCommand(Command) & 10 & Command + Undo \\
\hline util.StorableOutput.space() & 8 & Consistent behavior \\
\hline
\end{tabular}




\section{E. Non-seed methods for JHOTDRAW}

\section{List of the non-seed methods for JHotDraw}

Note: Several methods in this list could be considered aspect seeds. For instance, the "moveBy(..)" method could be considered a seed for the "move figure" concern. The analysis of such methods is an ongoing work.

\begin{tabular}{|c|c|}
\hline Qualified name (CH.ifa.draw.*) & Fan-in \\
\hline contrib.ComponentFigure.displayBox & 48 \\
\hline contrib.CustomToolBar.add & 16 \\
\hline contrib.DiamondFigure.containsPoint & 15 \\
\hline contrib.GraphicalCompositeFigure.displayBox & 49 \\
\hline contrib.html.HTMLTextAreaFigure.containsPoint & 15 \\
\hline contrib.html.HTMLTextAreaFigure.moveBy & 12 \\
\hline contrib.PolygonFigure.containsPoint & 15 \\
\hline contrib.PolygonFigure.displayBox & 48 \\
\hline contrib.PolygonFigure.pointCount & 12 \\
\hline contrib.TextAreaFigure.displayBox & 53 \\
\hline contrib.TextAreaFigure.moveBy & 12 \\
\hline contrib.TriangleFigure.containsPoint & 15 \\
\hline contrib.zoom.ScalingGraphics.fillRect & 11 \\
\hline figures.BorderDecorator.displayBox & 48 \\
\hline figures.ElbowConnection.updateConnection & 11 \\
\hline figures.EllipseFigure.displayBox & 50 \\
\hline figures.GroupFigure.displayBox & 47 \\
\hline figures.ImageFigure.displayBox & 47 \\
\hline figures.LineConnection.updateConnection & 12 \\
\hline figures.NullFigure.displayBox & 47 \\
\hline figures.PolyLineFigure.containsPoint & 15 \\
\hline figures.PolyLineFigure.displayBox & 48 \\
\hline figures.PolyLineFigure.pointAt & 15 \\
\hline figures.RectangleFigure.displayBox & 53 \\
\hline figures.RoundRectangleFigure.displayBox & 52 \\
\hline figures.TextFigure.displayBox & 53 \\
\hline figures.TextFigure.moveBy & 12 \\
\hline framework.ConnectionFigure.updateConnection & 11 \\
\hline framework.DrawingView.addToSelection & 11 \\
\hline framework.DrawingView.clearSelection & 31 \\
\hline framework.DrawingView.selection & 17 \\
\hline framework.Figure.containsPoint & 15 \\
\hline framework.Figure.moveBy & 13 \\
\hline framework.Figure.size & 10 \\
\hline framework. Handle.displayBox & 12 \\
\hline standard.AbstractCommand.view & 46 \\
\hline standard.AbstractFigure.containsPoint & 16 \\
\hline standard.AbstractFigure.moveBy & 13 \\
\hline standard.AbstractFigure.size & 10 \\
\hline standard.AbstractHandle.displayBox & 12 \\
\hline standard.AbstractTool.drawing & 19 \\
\hline standard.NullDrawingView.selection & 14 \\
\hline standard.NullHandle.NullHandle & 11 \\
\hline standard.RelativeLocator.RelativeLocator & 10 \\
\hline standard.StandardDrawing.displayBox & 51 \\
\hline standard.StandardDrawingView.addToSelection & 11 \\
\hline standard.StandardDrawingView.selection & 16 \\
\hline util.CommandMenu.CommandMenu & 15 \\
\hline
\end{tabular}




\section{F. Utility methods for JHOTDRAW}

List of the utility methods for JHotDraw

\begin{tabular}{cc} 
Qualified name (CH.ifa.draw.*) & Fan-in \\
\hline framework.FigureEnumeration.hasNextFigure & 96 \\
framework.FigureEnumeration.nextFigure & 92 \\
standard.FigureAndEnumerator.hasNextFigure & 96 \\
standard.FigureAndEnumerator.nextFigure & 92 \\
standard.FigureEnumerator.FigureEnumerator & 26 \\
standard.FigureEnumerator.hasNextFigure & 96 \\
standard.FigureEnumerator.nextFigure & 92 \\
standard.HandleEnumerator.HandleEnumerator & 21 \\
standard.ReverseFigureEnumerator.hasNextFigure & 96 \\
standard.ReverseFigureEnumerator.nextFigure & 92 \\
standard.SingleFigureEnumerator.hasNextFigure & 96 \\
standard.SingleFigureEnumerator.nextFigure & 92 \\
standard.SingleFigureEnumerator.SingleFigureEnumerator & 20 \\
util.collections.jdk11.CollectionsFactoryJDK11.createList & 26 \\
util.collections.jdk11.IteratorWrapper.hasNext & 39 \\
util.collections.jdk11.IteratorWrapper.next & 39 \\
util.collections.jdk11.ListWrapper.contains & 12 \\
util.collections.jdk11.ListWrapper.iterator & 31 \\
util.collections.jdk11.ListWrapper.size & 38 \\
util.collections.jdk11.SetWrapper.iterator & 10 \\
util.CollectionsFactory.createList & 26 \\
util.CollectionsFactory.current & 26 \\
util.ReverseListEnumerator.hasNext & 81 \\
util.ReverseListEnumerator.next & 39 \\
& 39
\end{tabular}




\section{G. Selected seed methods for TOMCAT}

\section{List of the selected seed methods for Tomcat/Catalina}

Note: The analysis of the Tomcat/Catalina case-study is an ongoing work.

This list is not intended to be exhaustive or definitive but it is the result of a preliminary investigation.

\begin{tabular}{|c|c|c|}
\hline Qualified name (org.apache. ${ }^{*}$ ) & Fan-in & Aspect \\
\hline catalina.ant.AbstractCatalinaTask.execute() & 14 & $\begin{array}{l}\text { Contract enforcement } \\
\quad+\text { Command } \\
\text { Contract enforcement }\end{array}$ \\
\hline catalina.ant.AbstractCatalinaTask.execute(String) & 14 & + Command \\
\hline catalina.authenticator.AuthenticatorBase.stop() & 27 & Lifecycle \\
\hline catalina.authenticator.SingleSignOn.stop() & 26 & Lifecycle \\
\hline catalina.authenticator.AuthenticatorBase.start() & 26 & Lifecycle \\
\hline catalina.authenticator.SingleSignOn.start() & 25 & Lifecycle \\
\hline catalina.authenticator.SSLAuthenticator.stop() & 26 & Lifecycle \\
\hline catalina.authenticator.SSLAuthenticator.start() & 25 & Lifecycle \\
\hline & & $\begin{array}{c}\text { Contract enforcement/consistent } \\
\text { behavior }\end{array}$ \\
\hline $\begin{array}{l}\text { catalina.connector.ResponseFacade.isCommitted() } \\
\text { catalina.connector.HttpResponseFacade.sendError(..) }\end{array}$ & 55 & + Façade \\
\hline $\begin{array}{l}\text { - overloaded } \\
\text { catalina.connector.ResponseWriter.print } \ln (. .)\end{array}$ & $19 / 24$ & Façade \\
\hline - overlaoded & 88 & Wrapper \\
\hline catalina.connector.ResponseWriter.flush() & 12 & Wrapper \\
\hline $\begin{array}{c}\text { catalina.connector.HttpResponseFacade.addHeader } \\
\text { (String, String) } \\
\text { catalina.connector.ResponseWriter.print(..) }\end{array}$ & 10 & Façade \\
\hline $\begin{array}{l}\text { - overloaded } \\
\text { catalina core ContainerBase fireContainerEvent }\end{array}$ & 29 & Wrapper \\
\hline (String, Object) & 69 & Observer \\
\hline catalina.core.ContainerBase.stop() & 33 & Lifecycle \\
\hline catalina.core.StandardContext.stop() & 30 & Lifecycle \\
\hline catalina.core.ContainerBase.start() & 30 & Lifecycle \\
\hline catalina.core.StandardService.stop() & 29 & Lifecycle \\
\hline catalina.core.StandardContext.start() & 27 & Lifecycle \\
\hline catalina.core.StandardEngine.stop() & 27 & Lifecycle \\
\hline catalina.core.StandardWrapper.stop() & 27 & Lifecycle \\
\hline catalina.core.StandardServer.stop() & 26 & Lifecycle \\
\hline catalina.core.StandardService.start() & 26 & Lifecycle \\
\hline catalina.core.StandardPipeline.stop() & 26 & Lifecycle \\
\hline catalina.core.StandardServer.start() & 25 & Lifecycle \\
\hline catalina.core.StandardWrapper.start() & 25 & Lifecycle \\
\hline catalina.core.StandardPipeline.start() & 25 & Lifecycle \\
\hline catalina.core.StandardEngine.start() & 25 & Lifecycle \\
\hline catalina.core.ContainerBase.findChild(String) & 23 & Composite \\
\hline catalina.core.ContainerBase.findChildren() & 21 & Composite \\
\hline $\begin{array}{l}\text { catalina.core.ContainerBase.removeChild(Container) } \\
\text { catalina.core.ContainerBase.addLifecycleListener }\end{array}$ & 11 & Composite \\
\hline (LifecycleListener) & 11 & Observer + Lifecycle \\
\hline catalina.core.StandardValveContext.invokeNext & & \\
\hline (Request, Response) & 10 & Chain of responsibility \\
\hline catalina.core.ApplicationContext.log(String, Throwable) & 10 & Logging (for exception handling) \\
\hline catalina.core.StandardContext.removeChild(Container) & 10 & Composite \\
\hline catalina.realm.RealmBase.stop() & 32 & Lifecycle \\
\hline catalina.realm.RealmBase.start() & 31 & Lifecycle \\
\hline
\end{tabular}




\section{H. Selected seed methods for TOMCAT- cont.}

Qualified name (org.apache.*)

catalina.realm.JAASRealm.stop()

catalina.realm.JDBCRealm.stop()

catalina.realm.UserDatabaseRealm.stop()

catalina.realm.JNDIRealm.stop()

catalina.realm.MemoryRealm.stop()

catalina.realm.DataSourceRealm.stop()

catalina.realm.UserDatabaseRealm.start()

catalina.realm.JDBCRealm.start()

catalina.realm.JNDIRealm.start()

catalina.realm.DataSourceRealm.start()

catalina.realm.MemoryRealm.start()

catalina.realm.JAASRealm.start()

catalina.realm.RealmBase.log(..) - overloaded catalina.logger.FileLogger.log(String) catalina.logger.SystemOutLogger.log(String) catalina.logger.SystemErrLogger.log(String)

catalina.logger.LoggerBase.stop() catalina.logger.FileLogger.stop() catalina.logger.LoggerBase.start() catalina.logger.FileLogger.start()

catalina.loader.WebappClassLoader.stop() catalina.loader.WebappLoader.stop() catalina.loader.WebappLoader.start() catalina.loader.WebappClassLoader.start() catalina.valves.ExtendedAccessLogValve.stop() catalina.valves.JDBCAccessLogValve.stop() catalina.valves.AccessLogValve.stop() catalina valves. AccessLogValve start() catalina.valves. ExtendedAccessLogValve.start() catalina.valves.JDBCAccessLogValve.start()

Fan-in

26

26

\section{Aspect}

Lifecycle +

contract enforcement/consistent

behavior

Lifecycle +

contract enforcement/consistent

behavior

Lifecycle +

contract enforcement/consistent behavior Lifecycle +

contract enforcement/consistent behavior Lifecycle +

contract enforcement/consistent

behavior Lifecycle +

contract enforcement/consistent behavior Lifecycle +

contract enforcement/consistent behavior Lifecycle +

contract enforcement/consistent behavior Lifecycle +

contract enforcement/consistent

behavior

Lifecycle +

contract enforcement/consistent behavior Lifecycle +

contract enforcement/consistent behavior Lifecycle +

contract enforcement/consistent

behavior

Logging

Logging

Logging

Logging

Lifecycle

Lifecycle

Lifecycle

Lifecycle

Lifecycle

Lifecycle

Lifecycle

Lifecycle

Lifecycle

Lifecycle

Lifecycle

Lifecycle

Lifecycle

Lifecycle 


\section{Selected seed methods for TOMCAT- cont.}

\begin{tabular}{|c|c|c|}
\hline Qualified name (org.apache.*) & Fan-in & Aspect \\
\hline $\begin{array}{c}\text { catalina.util.LifecycleSupport.fireLifecycleEvent } \\
\text { (String, Object) }\end{array}$ & 43 & Observer (+ Lifecycle) \\
\hline catalina.util.LifecycleSupport.addLifecycleListener & & \\
\hline $\begin{array}{l}\text { (LifecycleListener) } \\
\text { catalina.util.LifecycleSupport.removeLifecycleListener }\end{array}$ & 20 & Observer (+ Lifecycle) \\
\hline (LifecycleListener) & 20 & Observer (+ Lifecycle) \\
\hline catalina.util.LifecycleSupport.findLifecycleListeners() & 16 & Observer (+ Lifecycle) \\
\hline catalina.startup.Catalina.stop() & 29 & Lifecycle \\
\hline catalina.startup.Embedded.stop() & 29 & Lifecycle \\
\hline catalina.startup.Catalina.start() & 26 & Lifecycle \\
\hline catalina.startup.Embedded.start() & 26 & Lifecycle \\
\hline catalina.session.StoreBase.stop() & 27 & Lifecycle \\
\hline catalina.session.JDBCStore.stop() & 26 & Lifecycle \\
\hline catalina.session.PersistentManagerBase.stop() & 26 & Lifecycle \\
\hline catalina.session.StandardManager.stop() & 26 & Lifecycle \\
\hline catalina.session.StoreBase.start() & 26 & Lifecycle \\
\hline catalina.session.PersistentManagerBase.start() & 25 & Lifecycle \\
\hline catalina.session.StandardManager.start() & 25 & Lifecycle \\
\hline catalina.session.JDBCStore.start() & 25 & Lifecycle \\
\hline catalina.session.StoreBase.log(String) & 13 & Logging \\
\hline catalina.Lifecycle.stop() & 34 & Lifecycle (secondary interface) \\
\hline catalina.Lifecycle.start() & 28 & Lifecycle (secondary interface) \\
\hline catalina.Session.isValid() & 26 & Session management \\
\hline catalina.session.StandardSession.isValid() & 26 & Session management \\
\hline catalina.Container.findChild(String) & 23 & Composite \\
\hline catalina.Container.findChildren() & 21 & Composite \\
\hline catalina.Lifecycle.addLifecycleListener(LifecycleListener) & 12 & Observer (+ Lifecycle) \\
\hline catalina.ServerFactory.getServer() & 11 & Singleton \\
\hline catalina.Container.removeChild(Container) & 10 & Composite \\
\hline catalina.ValveContext.invokeNext(Request, Response) & 10 & Chain of responsibility \\
\hline ajp.tomcat4.Ajp13Connector.stop() & 26 & Lifecycle \\
\hline ajp.tomcat4.Ajp13Processor.stop() & 26 & Lifecycle \\
\hline ajp.tomcat4.Ajp13Processor.start() & 25 & Lifecycle \\
\hline ajp.tomcat4.Ajp13Connector.start() & 25 & Lifecycle \\
\hline ajp.tomcat4.config.BaseJkConfig.log(String) & 13 & Logging \\
\hline coyote.tomcat5.CoyoteResponseFacade.isCommitted() & 39 & Consistent behavior (+ façade) \\
\hline coyote.tomcat4.CoyoteResponseFacade.isCommitted() & 39 & Consistent behavior (+ façade) \\
\hline coyote.tomcat5.CoyoteConnector.stop() & 27 & Lifecycle \\
\hline coyote.tomcat4.CoyoteConnector.stop() & 26 & Lifecycle \\
\hline coyote.tomcat5.CoyoteConnector.start() & 25 & Lifecycle \\
\hline coyote.tomcat4.CoyoteConnector.start() & 25 & Lifecycle \\
\hline coyote.tomcat4.CoyoteResponseFacade.sendError(..) & & \\
\hline $\begin{array}{c}\text { - overloaded } \\
\text { coyote.tomcat5.CoyoteResponseFacade.sendError(..) }\end{array}$ & 24 & Façade \\
\hline - overloaded & 24 & Façade \\
\hline coyote.tomcat4.CoyoteResponse.isCommitted() & 23 & $\begin{array}{l}\text { Consistent behavior } \\
\text { Logging }\end{array}$ \\
\hline jk.config.BaseJkConfig.log(String) & 13 & (debugging, exception handling) \\
\hline naming.resources.ResourceAttributes.put(String, Object) & 10 & Consistent behavior \\
\hline tomcat.util.log.Log.isDebugEnabled() & 173 & Logging \\
\hline tomcat.util.log.Log.error(..) - overloaded & 128 & Logging \\
\hline $\begin{array}{l}\text { tomcat.util.compat.JdkCompat.chainException } \\
\text { (Throwable, Throwable) }\end{array}$ & 34 & $\begin{array}{c}\text { Exception handling } \\
\text { (consistent behavior/contract } \\
\text { enforcement) }\end{array}$ \\
\hline
\end{tabular}


J. Selected seed methods for TOMCAT- cont.

Qualified name (org.apache.*)

tomcat.util.compat.Jdk14Compat.chainException (Throwable, Throwable)

tomcat.util.log.Log.isTraceEnabled()

tomcat.util.log.Log.info(..) - overloaded

tomcat.util.log.Log.debug(..) - overloaded

tomcat.util.log.Log.warn(..) - overloaded
Fan-in

Aspect

Exception handling

(consistent behavior/contract

enforcement)

Logging

Logging - debugging

Logging - warning 